\title{
LncRNA MVIH knockdown inhibits the malignancy progression through downregulating miR-505 mediated HMGB1 and CCNE2 in acute myeloid leukemia
}

\author{
Shandong $\mathrm{Ke}^{1}$, Xiaofen Zhou ${ }^{2}$ \\ ${ }^{1}$ Department of Hematology, Huangshi Central Hospital, Huangshi 435000, China; ${ }^{2}$ Department of Hematology, Xiangyang Central Hospital, \\ Affiliated Hospital Of Hubei University of Arts and Science, Xiangyang 441021, China \\ Contributions: (I) Conception and design: X Zhou; (II) Administrative support: None; (III) Provision of study materials or patients: S Ke; \\ (IV) Collection and assembly of data: All authors; (V) Data analysis and interpretation: All authors; (VI) Manuscript writing: All authors. \\ (VII) Final approval of manuscript: All authors. \\ Correspondence to: Xiaofen Zhou. Department of Hematology, Xiangyang Central Hospital, Xiangyang Central Hospital, Affiliated Hospital Of Hubei \\ University of Arts and Science, 136 Jingzhou Street, Xiangyang 441021, China. Email: xunsongweipq@163.com.
}

Background: This study aimed to investigate the regulatory role of long non-coding RNA associated with microvascular invasion in hepatocellular carcinoma $(\operatorname{lnc}-M V I H)$ in the progression of acute myeloid leukemia (AML) and the underlying mechanism.

Methods: $L n c-M V I H$ expression was detected in AML cell lines AML-193, KG-1, HL-60, OCI-AML2 and primary normal bone marrow mononuclear cells (BMMC). The effect of $l n c-M V I H$ knockdown on cell proliferation, apoptosis and miR-505 expression were detected by transfection of $\operatorname{lnc}-M V I H$ shRNA and control shRNA into KG-1 cells. And the effect of miR-505 knockdown on $l n c-M V I H$, cell proliferation, cell apoptosis as well as potential miR-505 target genes [high mobility group box 1 (HMGB1) and cyclin E2 (CCNE2)] in $l n c-M V I H$ knockdown treated KG-1 cells was assessed by transfection of $\operatorname{lnc}-M V I H$ shRNA and $l n c-M V I H$ shRNA \& miR-505 shRNA into KG-1 cells.

Results: $L n c-M V I H$ expression was elevated in AML-193, KG-1, OCI-AML2 cell lines, but similar in HL-60 cell line compared with primary normal BMMC. Lnc-MVIH knockdown inhibited cell proliferation but promoted cell apoptosis in KG-1 cells, meanwhile miR-505 expression was increased by $\operatorname{lnc}-M V I H$ knockdown in KG-1 cells. And in rescue experiments, miR-505 knockdown had no effect on expression of lnc-MVIH, while it increased the expressions of HMGB1 and CCNE2, promoted cell proliferation, inhibited cell apoptosis in $l n c-M V I H$ knockdown treated KG-1 cells.

Conclusions: $L n c-M V I H$ knockdown inhibits cell proliferation but promotes cell apoptosis via regulating miR-505 mediated HMGB1 and CCNE2 in AML.

Keywords: Acute myeloid leukemia (AML); apoptosis; long non-coding RNA associated with microvascular invasion in bepatocellular carcinoma (lnc-MVIH); miR-505; proliferation

Submitted Apr 03, 2019. Accepted for publication Sep 12, 2019.

doi: $10.21037 /$ tcr.2019.10.12

View this article at: http://dx.doi.org/10.21037/tcr.2019.10.12

\section{Introduction}

Acute myeloid leukemia (AML) is a hematological malignancy characterized by malignant transformation of clonal neoplastic hematopoietic stem cells as well as the impaired normal hematopoiesis in the bone marrow and peripheral blood (1). Current therapies for AML commonly consist of intensive induction chemotherapy, stem cell transplantation and post-remission consolidation, which contributes to the complete remission of approximately $50-70 \%$ of adults with AML. However, only $20 \%$ of 
patients with AML enjoy long-term disease-free survival, and refractory or relapsed AML is the main cause of AML mortality $(2,3)$. Existing evidence indicates that insight into genetic background of AML that foster the extensive understanding of AML pathology might lead to development of specific targeted therapy $(4,5)$. Therefore, it is necessary to investigate the mechanism underlying the development and progression of AML to explore new therapeutic strategies.

Long non-coding RNAs (lncRNA) are defined as RNAs longer than 200 nucleotides, which participate in multiple stages of gene regulation including chromatin modification, regulation of protein modification, chromatin structure, and so on $(6,7)$. Increasing number of researches reveal that lncRNAs also play important roles in diverse human diseases, especially cancers, and some lncRNAs, such as lnc-CCAT1, lnc-HOTAIR, are reported to exert functions through interacting with microRNA (miRNA) at the posttranscriptional level in AML $(6,8,9)$. Recently, increasing studies indicate that $\operatorname{lncRNA}$ associated with microvascular invasion in hepatocellular carcinoma $($ lnc-MVIH) is upregulated and participates in the progression and development of several solid cancers, such as breast cancer, non-small cell lung cancer and hepatocellular carcinoma (10-13). However, little is known about its role in hematological malignancies. In addition, $l n c-M V I H$ contains target sites for $m i R-505$ as retrieved from miRanda database analysis (www.miranda. org), which is a tumor suppressor in several malignancies including chronic myeloid leukemia (14-16). Therefore, we hypothesized that $l n c-M V I H$ might interact with miR-505 as well as $m i R-505$ downstream target genes and regulate AML progression. In the present study, we investigated the regulatory role of $\operatorname{lnc}-M V I H$ in AML progression and the underlying mechanism, aiming to provide a novel insight of potential therapeutic targets in AML $(17,18)$.

\section{Methods}

\section{Cell sources and culture}

Human AML cell lines AML-193, KG-1, HL-60 and OCIAML2 were purchased from Leibniz Institute DSMZGerman Collection of Microorganisms and Cell Cultures (Braunschweig, German). AML-193 cells were cultured in 90\% Iscove's Modified Dulbecco's Medium (Gibco, USA) and $10 \%$ fetal bovine serum (Gibco, USA); KG-1 and HL60 cells were cultured in 90\% Roswell Park Memorial Institute (RPMI) 1640 Medium (Gibco, USA) and 10\% fetal bovine serum (Gibco, USA); OCI-AML2 were cultured in 90\% Minimum Essential Medium (MEM) (Gibco, USA) and $10 \%$ fetal bovine serum (Gibco, USA).

\section{Lnc-MVIH expression in AML cell lines and primary normal bone marrow mononuclear cells (BMMC)}

Lnc-MVIH expression in AML cell lines AML-193, KG-1, HL-60 and OCI-AML2 was detected using real-time quantitative polymerase chain reaction (RT-qPCR). Besides, primary normal human BMMC was purchased from American type culture collection (Manassas, USA) and lnc-MVIH in BMMC was detected by RT-qPCR as control.

\section{Lnc-MVIH expression in $C D 34^{+} A M L$ cells and wild $A M L$ cell lines}

CD34 ${ }^{+}$AML cells were separated from wild AML cell lines (AML-193, KG-1, HL-60, OCI-AML2) using Dynabeads CD $34^{+}$Positive Isolation Kit (Thermo, USA) according to the instructions of the manufacture, and $\operatorname{lnc}-M V I H$ expression in $\mathrm{CD} 34^{+}$AML cells and wild AML cells was detected using RT-qPCR.

\section{Effect of lnc-MVIH knockdown on cell proliferation, apoptosis and potential target miR-505}

Control shRNA [Control(-) group] and $l n c-M V I H$ shRNA [lnc-MVIH(-) group] were constructed by Shanghai GenePharma Bio-Tech Company (Shanghai, China) using pGPH1 vector and transfected into KG-1 cells. $L n c-M V I H$ expression was detected at $24 \mathrm{~h}$ by RT-qPCR, cell proliferation was detected at $0,24,48$, and $72 \mathrm{~h}$ by Counting Kit-8 (CCK-8) (Dojindo, Japan), cell apoptosis rate was detected at $24 \mathrm{~h}$ by Annexin V-FITC Apoptosis Detection Kit (BD, USA), potential target $m i R-505$ was predicted using miRanda database analysis (www.miranda. org) and detected at $24 \mathrm{~h}$ by RT-qPCR, respectively.

\section{Effect of miR-505 knockdown on attenuating lnc-MVIH knockdown functions}

Lnc-MVIH shRNA [Lnc-MVIH(-) group], and $\operatorname{lnc}-M V I H$ shRNA plus miR-505 shRNA [Lnc-MVIH(-)\&miR-505(-) group] were constructed by Shanghai GenePharma BioTech Company (Shanghai, China) using pGPH1 vector and transfected into KG-1 cells. $L n c-M V I H$ and miR-505 expressions were detected at $24 \mathrm{~h}$ by RT-qPCR, miR-505 
Table 1 Primers applied in qPCR

\begin{tabular}{lll}
\hline Gene & Forward primer (5'-3') & Reverse primer (5'-3') \\
\hline LnC-MVIH & AATTTTGCACATCTGAACAGCC & TTCAAAATCCCACTACGCCCA \\
HMGB1 & TTCTTCCTCTTCTGCTCTGAGTATC & TTCATAAGGCTGCTTGTCATCTG \\
CCNE2 & GCTGCTGCCTTGTGCCATT & GTGCTCTTCGGTGGTGTCATAA \\
GAPDH & GACCACAGTCCATGCCATCAC & ACGCCTGCTTCACCACCTT \\
MiR-505 & ACACTCCAGCTGGGGGGAGCCAGGAAGTAT & TGTCGTGGAGTCGGCAATTC \\
U6 & CGCTTCGGCAGCACATATACTA & ATGGAACGCTTCACGAATTTGC \\
\hline
\end{tabular}

Table 2 Antibodies applied in Western blot

\begin{tabular}{lll}
\hline Antibody & Company & Dilution \\
\hline Primary antibody & & \\
Rabbit monoclonal to HMGB1 & Abcam (UK) & $1: 2,000$ \\
Rabbit monoclonal to Cyclin E2 & Abcam (UK) & $1: 2,000$ \\
Rabbit monoclonal to GAPDH & Abcam (UK) & $1: 3,000$ \\
Secondary antibody & & \\
Goat anti-rabbit IgG H\&L (HRP) & Abcam (UK) & $1: 5,000$ \\
\hline
\end{tabular}

target genes including bigh mobility group box 1 (HMGB1) and cyclin E2 (CCNE2) were detected at $24 \mathrm{~h}$ by RT-qPCR and Western Blot, cell proliferation was detected at 0, 24, 48, and 72 h by CCK-8 (Dojindo, Japan), cell apoptosis rate was detected at $24 \mathrm{~h}$ by Annexin V-FITC Apoptosis Detection Kit (BD, USA).

\section{$R T-q P C R$}

Total RNA was extracted from cells using TRIzol ${ }^{\mathrm{TM}}$ Reagent (Invitrogen, USA) and then reversely transcribed to cDNA using RT-PCR Quick Master Mix (Toyobo, Japan). Following that, RT-qPCR was performed using SYBR $^{\circledR}$ Green Realtime PCR Master Mix (Toyobo, Japan) to quantify $\operatorname{lnc}$-MVIH, miR-505, HMGB1 and CCNE2 expressions. The result was calculated using $2^{-\Delta \Delta C t}$ method with GAPDH as an internal reference. The primers used in RT-qPCR were listed in Table 1.

\section{Western blot}

Total protein was extracted with RIPA Buffer (Sigma, USA). The protein concentration in each sample was then measured using the Pierce ${ }^{\mathrm{TM}}$ Rapid Gold BCA Protein
Assay Kit (Thermo, USA). Twenty ug protein samples were added to NuPAGE ${ }^{\mathrm{TM}} 4-12 \%$ Bis-Tris Protein Gels (Thermo, USA) and transferred onto polyvinylidene fluoride membrane (Millipore, German). After blocking with BSA (Thermo, USA) for $2 \mathrm{~h}$, the membranes were incubated with the primary antibodies overnight at $4{ }^{\circ} \mathrm{C}$. Then, the membranes were incubated with the secondary antibody for $90 \mathrm{~min}$ at $37^{\circ} \mathrm{C}$. EasyBlot ECL kit (Sangon, China) was used to illuminized the bands and $\mathrm{X}$-ray film (Kodak, USA) was used to visualize the result. The antibodies used in this study were summarized in Table 2.

\section{Statistics}

GraphPad 7.01 (GraphPad Int., USA) was used for statistical analysis and drawing images. Data were mainly presented as mean \pm standard deviation, and compared by One-way ANOVA test followed by multiple comparisons test or $t$ test. $\mathrm{P}<0.05$ was considered as significant.

\section{Results}

The relative expression of lnc-MVIH in AML cell lines and primary normal $B M M C$

The relative expression of $\operatorname{lnc}-M V I H$ was elevated in AML193 ( $\mathrm{P}<0.05)$, KG-1 ( $<<0.001)$, OCI-AML2 ( $<<0.001)$ cells, but similar in HL-60 ( $\mathrm{P}>0.05)$ cells compared with primary normal BMMC (Figure 1), indicating that $\operatorname{lnc}-M V I H$ was upregulated in AML cell lines.

\section{The relative expression of Inc-MVIH in $\mathrm{CD}_{3} 4^{+}$and wild AML cells}

The relative expression of $\operatorname{lnc}-M V I H$ was elevated in CD $34^{+}$ AML-193 cells compared with wild AML-193 cells $(\mathrm{P}<0.05)$, 


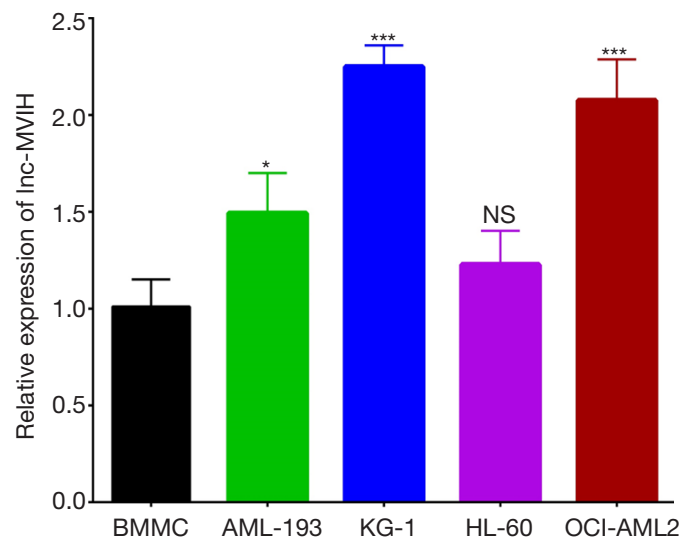

Figure $1 \mathrm{Lnc}-\mathrm{MVIH}$ expression in AML cells and primary normal BMMC. The comparison of $\operatorname{lnc}-M V I H$ expression between human AML cell lines and primary normal BMMC was detected by OneWay ANOVA followed by Dunnett's multiple comparisons test, which indicated that $\operatorname{lnc}-M V I H$ expression was elevated in AML193, KG-1 and OCI-AML2 cell lines, but similar in HL-60 cell lines compared to primary normal BMMC. $\mathrm{P}<0.05$ was considered significant. NS, non-significant; *, $\mathrm{P}<0.05$; ${ }^{* * *}, \mathrm{P}<0.001$. Lnc-MVIH, long non-coding RNA associated with microvascular invasion in hepatocellular carcinoma; AML, acute myeloid leukemia; BMMC, bone marrow mononuclear cells.

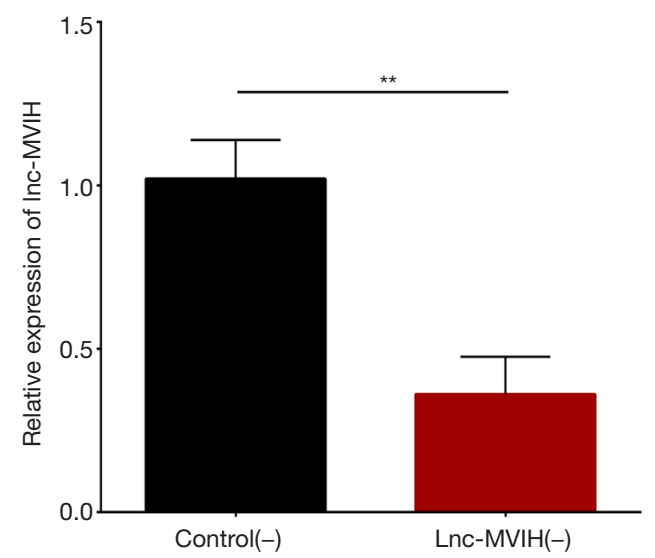

Figure 2 Lnc-MVIH knockdown decreased $\operatorname{lnc}-M V I H$ expression in KG-1 cells. $L n c-M V I H$ knockdown decreased $l n c-M V I H$ expression compared to control, which indicated the successful transfection. The comparison of $\operatorname{lnc}-M V I H$ expression between $\operatorname{lnc}-M V I H$ shRNA group and control was conducted by $t$-test. $\mathrm{P}<0.05$ was considered significant. ${ }^{* *}, \mathrm{P}<0.01$. Lnc-MVIH, long noncoding RNA associated with microvascular invasion in bepatocellular carcinoma. and was also increased in $\mathrm{CD} 34^{+} \mathrm{KG}-1$ cells compared with wild KG-1 cells $(\mathrm{P}<0.01)$, while similar between $\mathrm{CD} 34^{+}$ HL-60 cells and wild HL-60 cells $(\mathrm{P}>0.05)$, between CD34 ${ }^{+}$ OCI-AML2 cells and wild OCI-AML2 cells $(\mathrm{P}>0.05)$ (Figure S1).

\section{The relative expression of lnc-MVIH after transfection}

The expression of $\operatorname{lnc}-M V I H$ was decreased in $\operatorname{lnc}-M V I H(-)$ group compared with Control(-) group, which indicated the successful transfection of $\operatorname{lnc}-M V I H$ shRNA $(\mathrm{P}<0.01)$ (Figure 2).

\section{Effect of lnc-MVIH knockdown on cell proliferation and} apoptosis in KG-1 cells

Cell proliferation was decreased in $\operatorname{lnc}-M V I H(-)$ group at $48 \mathrm{~h}(\mathrm{P}<0.05)$ and $72 \mathrm{~h}(\mathrm{P}<0.05)$, but unchanged at $0 \mathrm{~h}(\mathrm{P}>0.05)$ and $24 \mathrm{~h}(\mathrm{P}>0.05)$ compared with Control(-) group (Figure $3 A$ ). Cell apoptosis rate was increased in $L n c-$ MVIH(-) group compared with Control(-) group at $24 \mathrm{~h}$ $(\mathrm{P}<0.01)$ (Figure 3B,C). These data suggested that $\operatorname{lnc}-M V I H$ knockdown suppressed cell proliferation but promoted cell apoptosis in AML.

The relative expression of miR-505 after transfection in KG-1 cells

The relative expression of $m i R-505$ was elevated in $l n c-$ $M V I H(-)$ group compared with Control(-) group after transfection $(\mathrm{P}<0.01)$, which indicated that $l n c-M V I H$ knockdown promoted miR-505 expression in AML (Figure 4).

The effect of miR-505 knockdown on HMGB1 and CCNE2 expressions in lnc-MVIH knockdown treated KG-1 cells

The rescue experiments were conducted to explore the effect of miR-505 knockdown on HMGB1 and CCNE2 expressions in lnc-MVIH knockdown treated KG-1 cells. Firstly, we observed that the relative expression of $m i R$ 505 was decreased in $\operatorname{lnc}-M V I H(-) \& m i R-505(-)$ group compared with $\operatorname{lnc}-M V I H(-)$ group $(\mathrm{P}<0.01)$, which indicated the successful transfection of $m i R-505$ shRNA (Figure 5A). Then the relative expression of $\operatorname{lnc}-M V I H$ was 

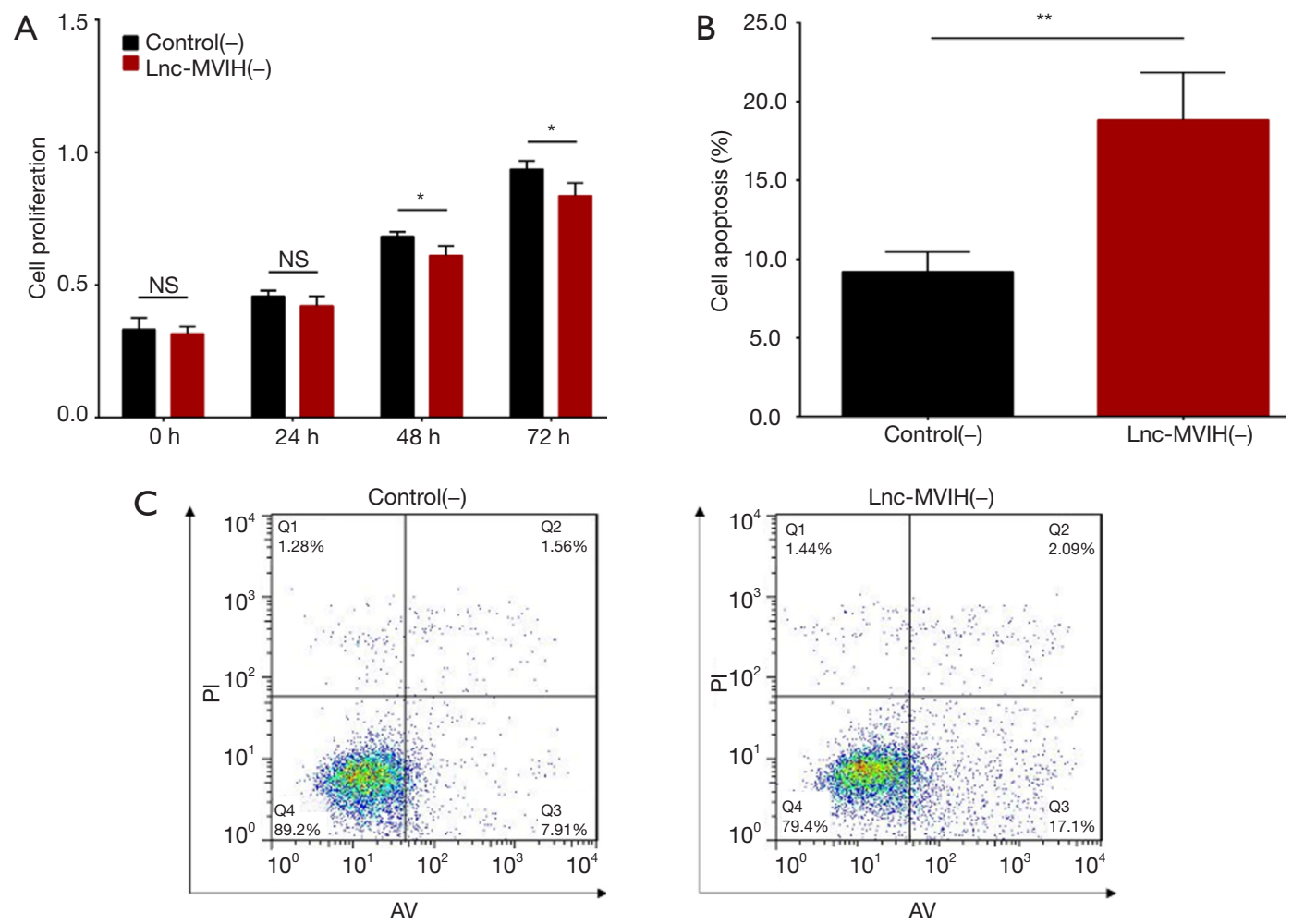

Figure $3 L n c-M V I H$ knockdown decreased cell proliferation but promoted cell apoptosis in KG-1 cells. Lnc-MVIH knockdown had no effect on cell proliferation at 0 and $24 \mathrm{~h}$ but decreased cell proliferation at 48 and $72 \mathrm{~h}$ (A). Lnc-MVIH knockdown increased cell apoptosis (B,C). $\mathrm{P}<0.05$ was considered significant. * $\mathrm{P}<0.05$. Lnc-MVIH, long non-coding RNA associated with microvascular invasion in hepatocellular carcinoma; NS, non-significant.

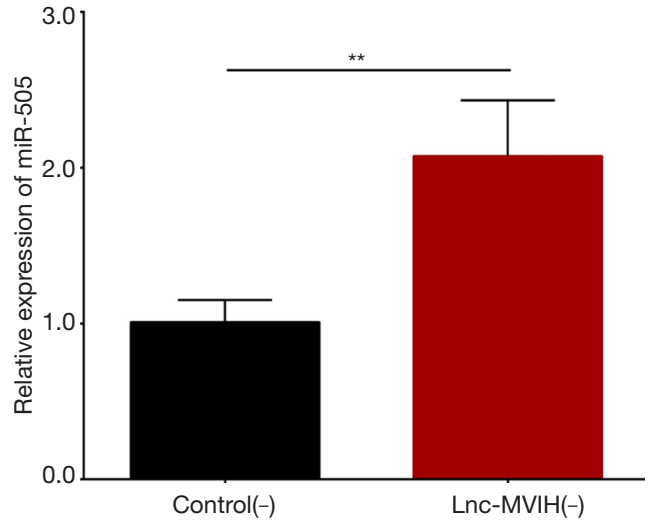

Figure $4 \mathrm{Lnc}-\mathrm{MVIH}$ knockdown increased miR-505 expression KG-1 cells. Lnc-MVIH knockdown increased miR-505 expression in AML. The comparison of $m i R-505$ expression between $l n c-$ $M V I H$ knockdown and control was conducted by $t$-test. $\mathrm{P}<0.05$ was considered significant. **, $\mathrm{P}<0.01$. Lnc-MVIH, long non-coding $R N A$ associated with microvascular invasion in hepatocellular carcinoma; AML, acute myeloid leukemia. shown to be similar in both $\operatorname{Lnc}-M V I H(-)$ \& miR-505(-) group and $L n c-M V I H(-)$ group $(\mathrm{P}>0.05)$, which suggested that $m i R-505$ did not regulate $l n c-M V I H$ (Figure $5 B$ ). Finally, $H M G B 1$ and $C C N E 2$, as the target genes of $m i R-505$, were detected, which exhibited that the relative expressions of HMGB1 $(\mathrm{P}<0.05)$ (Figure $5 C)$ and CCNE2 $(\mathrm{P}<0.05)$ (Figure 5D) were increased in $\operatorname{lnc}-M V I H(-) \& m i R-505(-)$ group compared with $\operatorname{lnc}$ - MVIH(-) group. And Western blot also visualized that $H M G B 1$ and $C C N E 2$ protein expression was increased in $\operatorname{lnc}$-MVIH(-) \& miR-505(-) group compared with $\operatorname{lnc}-M V I H(-)$ group (Figure $5 E$ ). These data suggested that $m i R-505$ knockdown increased the expressions of $H M G B 1$ and $C C N E 2$ in $\operatorname{lnc}-M V I H$ knockdown treated AML cells.

\section{The effect of miR-505 knockdown on cell proliferation and cell apoptosis in lnc-MVIH knockdown treated KG-1 cells}

Cell proliferation was increased in $\operatorname{lnc}-M V I H(-) \& m i R-$ 

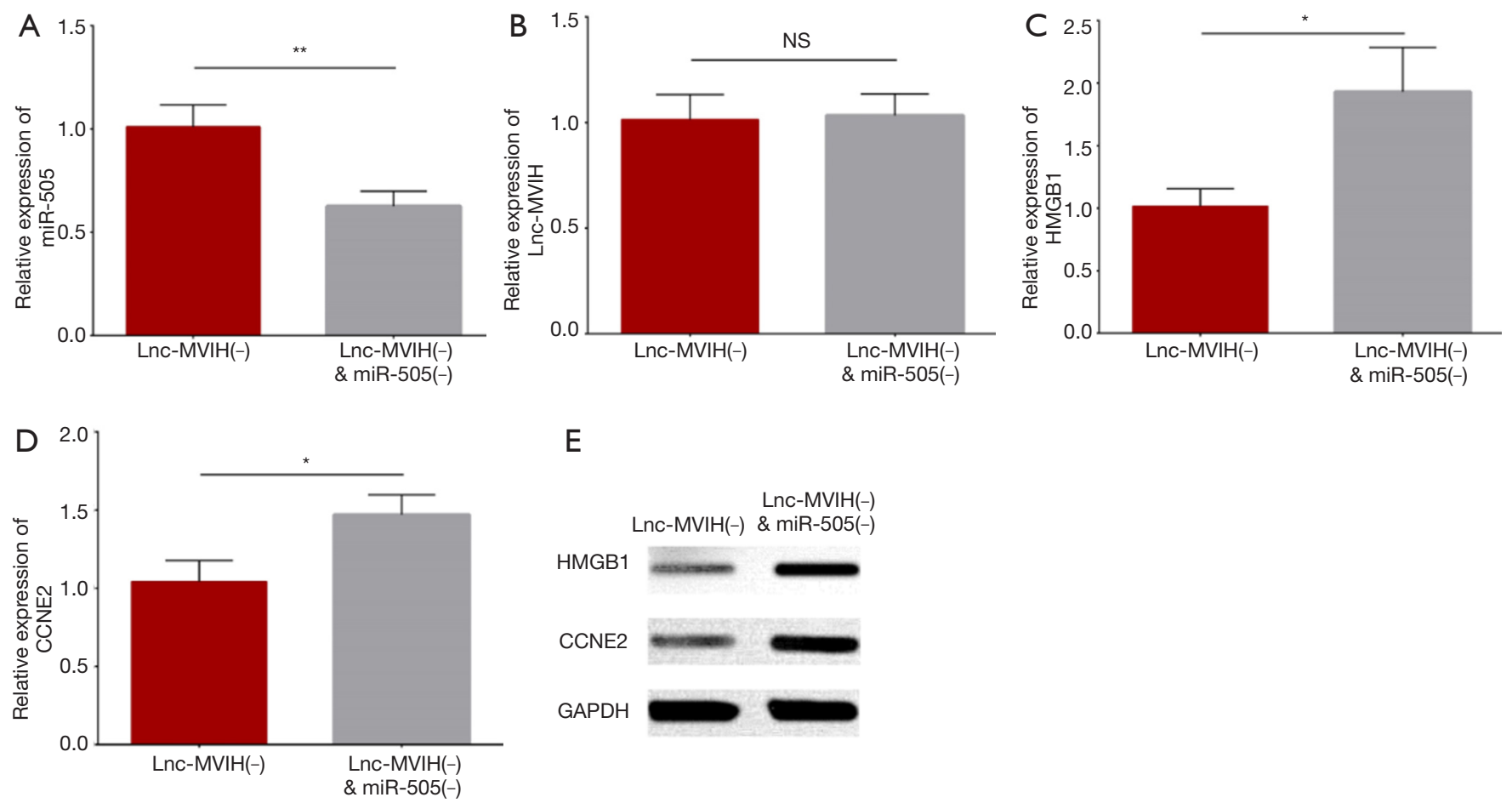

$\mathrm{E}$

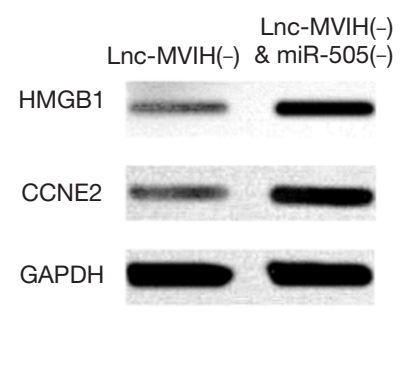

Figure 5 MiR-505 knockdown increased HMGB1 and CCNE2 expressions in $l n c-M V I H$ knockdown treated KG-1 cells. Lnc-MVIH(-) \& $m i R-505(-)$ group presented decreased expression of miR-505 compared to $l n c-M V I H(-)$ group (A). Lnc-MVIH(-) \& miR-505(-) group and $\operatorname{lnc}-M V I H(-)$ group showed the similar expression of $l n c-M V I H(\mathrm{~B}) . \operatorname{Lnc}-M V I H(-) \& m i R-505(-)$ group exhibited increased mRNA and protein expressions of HMGB1 compared to $\operatorname{lnc}-M V I H(-)$ group (C,E). Lnc-MVIH(-) \& miR-505(-) group also displayed increased mRNA and protein expressions of $C C N E 2$ compared to $\operatorname{lnc}-M V I H(-)$ group $(\mathrm{D}, \mathrm{E}) . \mathrm{P}<0.05$ was considered significant. *, $\mathrm{P}<0.05 ;$ **, $\mathrm{P}<0.01$. Lnc$M V I H$, long non-coding RNA associated with microvascular invasion in bepatocellular carcinoma; lnc-MVIH(-), lnc-MVIH shRNA; lnc-MVIH(-) \& miR-505(-), lnc-MVIH shRNA plus miR-505 shRNA; NS, non-significant.

$505(-)$ group at $48 \mathrm{~h}(\mathrm{P}<0.05)$ and $72 \mathrm{~h}(\mathrm{P}<0.01)$, but unchanged at $0 \mathrm{~h}(\mathrm{P}>0.05)$ and $24 \mathrm{~h}(\mathrm{P}>0.05)$ compared with $\operatorname{lnc}-M V I H(-)$ group (Figure $6 A$ ). Cell apoptosis rate was decreased in $\operatorname{lnc}-M V I H(-) \& m i R-505(-)$ group compared with $\operatorname{lnc}-M V I H(-)$ group at $24 \mathrm{~h}(\mathrm{P}<0.01)$ (Figure 6B,C). These data displayed that $m i R-505$ knockdown compensated the effect of $\operatorname{lnc}-M V I H$ knockdown on cell proliferation and cell apoptosis in AML. Combining all the data above, we speculated that $l n c-M V I H$ knockdown inhibited AML progression possibly via regulating miR-505 mediated HMGB1 and CCNE2 in AML.

\section{Discussion}

In the present study, we observed that $\operatorname{lnc}-M V I H$ was upregulated in AML cell lines compared with primary normal BMMC, and its knockdown suppressed cell proliferation but promoted cell apoptosis via regulating
miR-505 mediated HMGB1 and CCNE2 in AML.

Lnc-MVIH is located in the intron of the RPS24 gene and is essential for transcription process of RPS24 (19). Existing studies demonstrate that it is upregulated and closely related with tumorigenesis and metastasis in some solid tumors $(11-13,19)$. Lnc-MVIH is initially found to be upregulated in hepatocellular carcinoma and promotes tumor growth and metastasis by activating tumorinducing angiogenesis (10). It is also indicated to be overexpressed in MDA-MB-231 breast cancer cell line compared to mammary epithelial cells and lnc-MVIH knockdown inhibits cell proliferation, increases cell apoptosis, elevates the G1-G0 phase cell proportion and decreases the percentage of cells in the $\mathrm{S}$ phase in MDAMB-231 breast cancer cell line (13). In another study, $l n c$ $M V I H$ is upregulated and its knockdown impaires cell proliferation as well as invasion in non-small cell lung cancer (12). Furthermore, the insufficiency of RPS24 gene, 

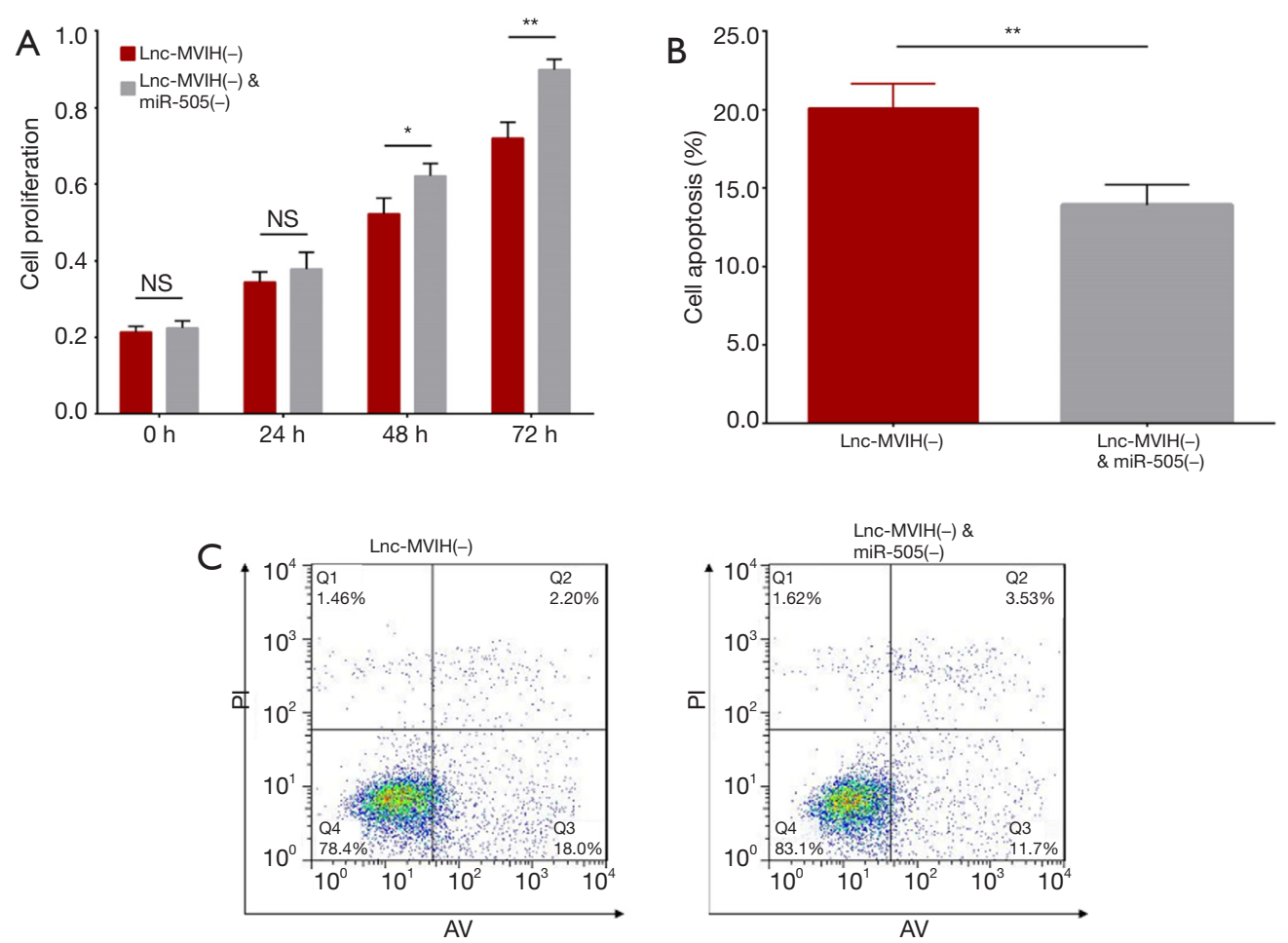

Figure 6 MiR-505 knockdown promoted cell proliferation but inhibited cell apoptosis in $\operatorname{lnc}$-MVIH knockdown treated KG-1 cells. Lnc$M V I H(-) \& m i R-505(-)$ group presented increased cell proliferation at 48 and $72 \mathrm{~h}$, but unchanged at 0 and $24 \mathrm{~h}$ compared to lnc-MVIH(-) group (A). Lnc-MVIH(-) \& $m i R-505(-)$ group showed decreased cell apoptosis compared to $\operatorname{lnc}-M V I H(-)$ group (B, C). $\mathrm{P}<0.05$ was considered significant. *, $\mathrm{P}<0.05$; **, $\mathrm{P}<0.01$. Lnc-MVIH, long non-coding RNA associated with microvascular invasion in bepatocellular carcinoma; lnc-MVIH(-), lnc-MVIH shRNA; Lnc-MVIH(-) \& miR-505(-), lnc-MVIH shRNA plus miR-505 shRNA; NS, non-significant.

where $\operatorname{lnc}-M V I H$ is located on, is indicated to cause the cell cycle defects in hematological diseases such as diamondBlackfan anemia (20). In addition, through the miRanda database analysis we found that $\operatorname{lnc}-M V I H$ contained several target sites of $m i R-505$ which was shown to be biomarkers in chronic myeloid leukemia (20). Therefore, we hypothesized that $\operatorname{lnc}-M V I H$ might participate in the biological processes of AML. In our study, we observed that lnc-MVIH was overexpressed in AML cell lines compared with primary normal BMMC, and lnc-MVIH knockdown inhibited cell proliferation but promoted cell apoptosis in AML. The possible reasons might include that (I) $\operatorname{lnc}-M V I H$ might serve as the sponge for miRNA (such as miR-505), leading to the inhibitory effect on miRNA target genes (such as HMGB1 and CCNE2), contributing to the AML progression (16). (II) Lnc-MVIH knockdown might inactivate the oncogenic pathways such as WNT signaling pathway to inhibit the malignant progression (21).

Existing evidence exhibits that $\operatorname{lnc}-M V I H$ is involved in the regulation of miRNA target genes via serving as miRNA sponge in solid tumors (6). For example, $l n c-$ $M V I H$ knockdown inhibits the cell viability and promotes cell apoptosis through upregulating $m i R-199 a$ and $m i R$ $199 a$ targeted FZD7 in hepatocellular carcinoma (11). In addition, miRanda database analysis reveals that $m i R$ 505, which suppresses cell proliferation in hematological malignancies, is the potential target of $\operatorname{lnc}-M V I H(15,16,22)$. Then we detected the expression of $m i R-505$ after transfecting $\operatorname{lnc}-M V I H$ shRNA into KG-1 cells and found that $\operatorname{lnc}-M V I H$ knockdown upregulated the expression of miR-505. Furthermore, HMGB1 and CCNE2 are potential target genes of $m i R-505$, which are previously shown to be oncogenes in hematological malignancies $(22,23)$. HMGB1, as a common oncogene in $\mathrm{AML}$, triggers pro-inflammatory stimulus and activates the immune receptors, leading to the proliferation of AML cells, which is critical for the progression of AML $(18,23,24)$. Besides, CCNE2 is often regarded as a key gene involved in cell cycle control, whose 
overexpression results in the proliferation advantage and malignancy progression in AML $(17,25)$. Therefore, we conducted the rescue experiments to verify whether $m c^{-}$ $M V I H$ knockdown repressed cell activities via regulating miR-505 mediated HMGB1 and CCNE2, and found that $m i R-505$ knockdown increased the expression of HMGB1 and CCNE2 in $l n c-M V I H$ knockdown treated AML cells, meanwhile miR-505 knockdown compensated the effect of lnc-MVIH knockdown on cell proliferation and cell apoptosis in $l n c-M V I H$ knockdown treated AML cells. Based on these data, we summarized that $m c-M V I H$ knockdown inhibited cell proliferation but promoted cell apoptosis via regulating miR-505 mediated HMGB1 and CCNE2. The possible reasons might include that: (I) $\operatorname{lnc}-M V I H$ knockdown impaired the expression of cell cycle regulatory proteins in the oncogenic pathways (such as Akt pathway, which CCNE2 participated in) via upregulation of $m i R-505$, leading to the blocking effect of oncogenic pathway and inhibited cell proliferation but promoted cell apoptosis in AML (17). (II) $L n c-M V I H$ knockdown reduced the expression of HMGB1 and inhibited pro-inflammatory stimulus, which decreased proliferation and survival of AML cells (18).

In conclusion, $\operatorname{lnc}-M V I H$ knockdown inhibits cell proliferation but promotes cell apoptosis via regulating $m i R$ 505 mediated HMGB1 and CCNE2 in AML, which suggested that $m c-M V I H$ might serve as a potential therapeutic target for AML.

\section{Acknowledgments}

Funding: None.

\section{Footnote}

Conflicts of Interest: All authors have completed the ICMJE uniform disclosure form (available at http://dx.doi. org/10.21037/tcr.2019.10.12). The authors have no conflicts of interest to declare.

Ethical Statement: The authors are accountable for all aspects of the work in ensuring that questions related to the accuracy or integrity of any part of the work are appropriately investigated and resolved. The study was conducted in accordance with the Declaration of Helsinki (as revised in 2013). The institutional ethical approval and individual informed consent were waived due to the nature of the study.
Open Access Statement: This is an Open Access article distributed in accordance with the Creative Commons Attribution-NonCommercial-NoDerivs 4.0 International License (CC BY-NC-ND 4.0), which permits the noncommercial replication and distribution of the article with the strict proviso that no changes or edits are made and the original work is properly cited (including links to both the formal publication through the relevant DOI and the license). See: https://creativecommons.org/licenses/by-nc-nd/4.0/.

\section{References}

1. Liao Q, Wang B, Li X, et al. miRNAs in acute myeloid leukemia. Oncotarget 2017;8:3666-82.

2. Tallman MS, Gilliland DG, Rowe JM. Drug therapy for acute myeloid leukemia. Blood 2005;106:1154-63.

3. Daver N, Cortes J. Molecular targeted therapy in acute myeloid leukemia. Hematology 2012;17 Suppl 1:S59-62.

4. Cancer Genome Atlas Research Network, Ley TJ, Miller C, et al. Genomic and epigenomic landscapes of adult de novo acute myeloid leukemia. $\mathrm{N}$ Engl J Med 2013;368:2059-74.

5. Schlenk RF. Post-remission therapy for acute myeloid leukemia. Haematologica 2014;99:1663-70.

6. Wei S, Wang K. Long noncoding RNAs: pivotal regulators in acute myeloid leukemia. Exp Hematol Oncol 2016;5:30.

7. Halley P, Kadakkuzha BM, Faghihi MA, et al. Regulation of the apolipoprotein gene cluster by a long noncoding RNA. Cell Rep 2014;6:222-30.

8. Chen L, Wang W, Cao L, et al. Long Non-Coding RNA CCAT1 Acts as a Competing Endogenous RNA to Regulate Cell Growth and Differentiation in Acute Myeloid Leukemia. Mol Cells 2016;39:330-6.

9. Xing CY, Hu XQ, Xie FY, et al. Long non-coding RNA HOTAIR modulates c-KIT expression through sponging miR-193a in acute myeloid leukemia. FEBS Lett 2015;589:1981-7.

10. Yuan SX, Yang F, Yang Y, et al. Long noncoding RNA associated with microvascular invasion in hepatocellular carcinoma promotes angiogenesis and serves as a predictor for hepatocellular carcinoma patients' poor recurrence-free survival after hepatectomy. Hepatology 2012;56:2231-41.

11. Shi $\mathrm{Y}$, Song Q, Yu S, et al. Microvascular invasion in hepatocellular carcinoma overexpression promotes cell proliferation and inhibits cell apoptosis of hepatocellular 
carcinoma via inhibiting miR-199a expression. Onco Targets Ther 2015;8:2303-10.

12. Nie FQ, Zhu Q, Xu TP, et al. Long non-coding RNA $\mathrm{MVIH}$ indicates a poor prognosis for non-small cell lung cancer and promotes cell proliferation and invasion. Tumour Biol 2014;35:7587-94.

13. Lei B, Xu SP, Liang XS, et al. Long non-coding RNA $\mathrm{MVIH}$ is associated with poor prognosis and malignant biological behavior in breast cancer. Tumour Biol 2016;37:5257-64.

14. Tian L, Wang ZY, Hao J, et al. miR-505 acts as a tumor suppressor in gastric cancer progression through targeting HMGB1. J Cell Biochem 2018. [Epub ahead of print].

15. Lu L, Zhang D, Xu Y, et al. miR-505 enhances doxorubicin-induced cytotoxicity in hepatocellular carcinoma through repressing the Akt pathway by directly targeting HMGB1. Biomed. Pharmacother 2018;104:613-21.

16. Ramachandran SS, Muiwo P, Ahmad HM, et al. miR-505$5 \mathrm{p}$ and miR-193b-3p: potential biomarkers of imatinib response in patients with chronic myeloid leukemia. Leuk Lymphoma 2017;58:1981-84.

17. Payton M, Coats S. Cyclin E2, the cycle continues. Int J Biochem Cell Biol 2002;34:315-20.

18. Yasinska IM, Goncalves Silva I, Sakhnevych SS, et al. High mobility group box 1 (HMGB1) acts as an "alarmin" to promote acute myeloid leukaemia progression. Oncoimmunology 2018;7:e1438109.

19. He Y, Meng XM, Huang C, et al. Long noncoding RNAs: Novel insights into hepatocelluar carcinoma. Cancer Lett 2014;344:20-27.

20. Badhai J, Fröjmark AS, J Davey E, et al. Ribosomal protein S19 and S24 insufficiency cause distinct cell cycle defects in Diamond-Blackfan anemia. Biochim Biophys Acta 2009;1792:1036-42.

21. Zhou HR, Fu HY, Wu DS, et al. Relationship between epigenetic changes in Wnt antagonists and acute leukemia. Oncol Rep 2017;37:2663-71.

22. Lu L, Qiu C, Li D, et al. MicroRNA-505 suppresses proliferation and invasion in hepatoma cells by directly targeting high-mobility group box 1 . Life Sci 2016;157:12-8.

23. Yu Y, Xie M, He YL, et al. Role of high mobility group box 1 in adriamycin-induced apoptosis in leukemia K562 cells. Ai Zheng 2008;27:929-33.

24. Tang D, Kang R, Zeh HJ 3rd, et al. High-mobility group box 1 and cancer. Biochim Biophys Acta 2010;1799:131-40.

25. Qian Z, Joslin JM, Tennant TR, et al. Cytogenetic and genetic pathways in therapy-related acute myeloid leukemia. Chem Biol Interact 2010;184:50-7.
Cite this article as: $\mathrm{Ke} \mathrm{S}$, Zhou X. LncRNA MVIH knockdown inhibits the malignancy progression through downregulating $m i R$ 505 mediated $H M G B 1$ and $C C N E 2$ in acute myeloid leukemia. Transl Cancer Res 2019;8(7):2526-2534. doi: 10.21037/ tcr.2019.10.12 


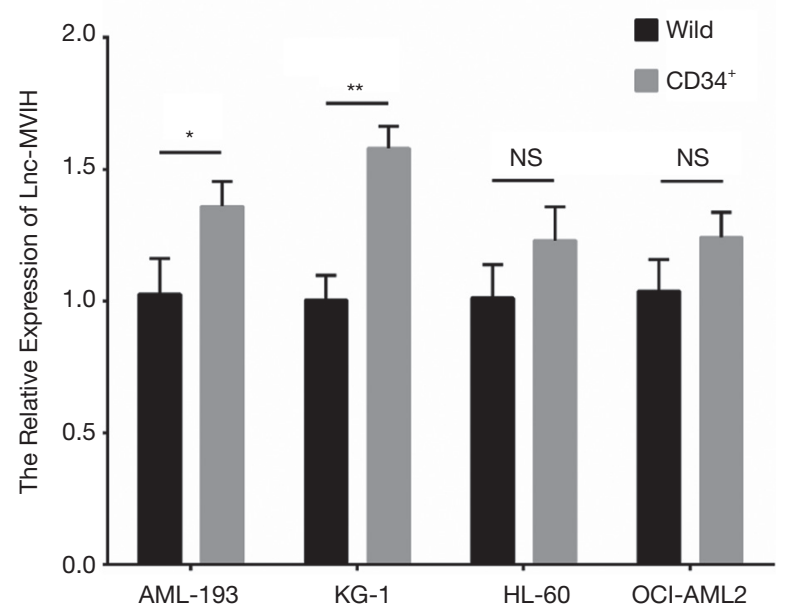

Figure S1 Lnc-MVIH expression in $\mathrm{CD}_{4} 4^{+}$and wild AML cells. The comparison of $l n c-M V I H$ expression between $\mathrm{CD} 34^{+}$and wild AML cells (AML-193, KG-1, HL-60 and OCL-AML2) was detected by $t$-test. $\mathrm{P}<0.05$ was considered significant. ${ }^{*}, \mathrm{P}<0.05 ;{ }^{* *}, \mathrm{P}<0.01$. LncMVIH, long non-coding RNA associated with microvascular invasion in bepatocellular carcinoma; AML, acute myeloid leukemia; NS, nonsignificant. 